\title{
Electron Avalanche Statistics
}

\author{
T. FICKER \\ Department of Physics, Brno University of Technology, Veveri 95, CZ-602 00 Brno, Czech Republic
}

(Received May 14, 2009; revised version August 11, 2009; in final form October 1, 2009)

\begin{abstract}
A solution of the long-lasting problem with anomalous population statistics of pre-streamer avalanches has been proposed in the form of a new generalized probability density function.
\end{abstract}

PACS numbers: 52.80.-s, 52.80.Dy, 52.80.Mg

\section{Introduction}

Since the times of the founders of streamer theory - Raether [1], Loeb [2] and Meek [3] — a special attention has been paid to high populated electron avalanches possessing potential to overgrowing into streamers. One of the basic problem that has not been resolved so far concerns anomalous statistical behavior of big avalanches with electron populations $n>10^{4}$. The mentioned anomaly consists in systematic deviations $[1,4,5]$ from the Furry/exponential distribution $[6,7], w(n)=1 /\langle n\rangle[1-1 /\langle n\rangle]^{n-1} \approx 1 /\langle n\rangle \exp (-n /\langle n\rangle)$, which has been considered as a general statistical law holding for all avalanches regardless of their size. Experimental evidence based on different testing methods [8-13] has identified the Pareto probability density function $w(n)=$ const $\cdot n^{-(1+D)}$ as a reliable distribution of high populated avalanches. However, this result would imply the existence of two independent statistics (Furry's and Pareto's) governing simultaneously one group of identical objects but such a dual concept is hardly acceptable. Here we show that there is a generalized statistical distribution which unifies both the mentioned statistics and in this way we remove the long-lasting problem of anomalous pre-streamer statistics. So far the anomalous statistics of big avalanches have been considered as experimental artifact [1]. Experimental evidence employing various experimental techniques [8-11] has shown that this is not the case and that the phenomenon has a real physical background. Our derivation of the generalized statistical distribution demonstrates how a special superposition of elementary functions may generate a new functional form showing perfect power law behavior, which is characteristic for fractal phenomena. This result has a broader meaning, namely, it supports the idea that fractal phenomena can result from a collective acting of more elementary processes that may be represented by properly chosen elementary functions. We anticipate that the new approach to the populations statistics of electron avalanches may assist in improving relevant parts of streamer theory since statistical behavior of pre-streamer avalanches inevitably determines the statistics of their streamer successors.

\section{Fractal multiplication of electron avalanches}

The Pareto statistics is a "typical fractal" statistics since it governs statistical features of all self-similar fractals with dimension $D$. This fact was taken into account when formulating the scenario of fractal avalanche multiplication [11]. The multiplication counts on displaced smaller avalanches that accompany big parent avalanches. The displaced avalanches are initiated by photoionization only after the parent avalanche has passed a certain critical distance $\bar{\Delta}$ long enough to create sufficiently intensive UV-radiation. On the track $\bar{\Delta}$ the parent avalanche accumulates a certain number of electrons $\bar{N}=\exp (\alpha \bar{\Delta})$ where $\alpha$ is the first Townsend ionization coefficient. The number of displaced avalanches is equal to the so-called "multiplicity" $\bar{K}$. As soon as the displaced avalanches overcome the critical distance $\bar{\Delta}$, they become parent avalanches for displaced avalanches of the second generation. The number of the displaced avalanches in the second generation is $\bar{K}^{2}$. In this way the process may continue to still higher generations $j$. It is the length $d$ of the discharge gap that limits the number of generations $J_{\max }=d / \bar{\Delta}-1$. But the process may also terminate before it reaches the last possible generation $J_{\max }$, i.e. $j=0,1,2, \ldots J \leq J_{\max }$. The probability density function of the $j$-th generation $w_{j}(n)=$ $\bar{K}^{j} /\left\langle n_{j}\right\rangle\left[1-1 /\left\langle n_{j}\right\rangle\right]^{n-1} \approx \bar{K}^{j} /\left\langle n_{j}\right\rangle \exp \left(-n /\left\langle n_{j}\right\rangle\right)$ possesses its own mean population $\left\langle n_{j}\right\rangle=\exp (\alpha(d-j \bar{\Delta}))$. Summing the probability density functions over all generations, a new probability function $F(n)$ may be found

$$
\begin{gathered}
F(n)=\frac{1}{\bar{n}_{d}} \sum_{j=0}^{J}(\bar{K} \bar{N})^{j}\left(1-\frac{\bar{N}^{j}}{\bar{n}_{d}}\right)^{n-1} \\
\approx \frac{1}{\bar{n}_{d}} \sum_{j=0}^{J}(\bar{K} \bar{N})^{j} \exp \left(-\frac{n \bar{N}^{j}}{\bar{n}_{d}}\right), \\
\bar{n}_{d}=\exp (\alpha d), \quad \bar{N} \geq 1, \quad \bar{K} \geq 1, \\
J \leq J_{\max }=\frac{d}{\bar{\Delta}}-1, \quad D=\frac{\ln \bar{K}}{\ln \bar{N}} .
\end{gathered}
$$

Since the whole process is highly stochastic, the mean values $\bar{n}_{d}, \bar{K}, \bar{N}$, and $\bar{\Delta}$ are employed. Function (1) com- 


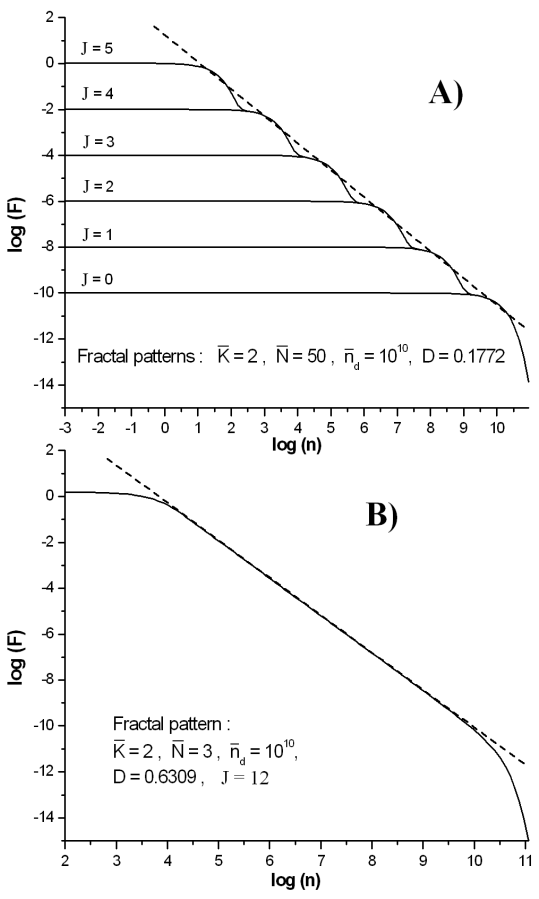

Fig. 1. Log-log plots of probability density function (1): (A) The "waves" on the graph represent generations of displaced avalanches. (B) "Unified waves" form a linear section which corresponds to power law behavior. After [11].

prises both the Furry and Pareto distributions as two special cases: taking $J=0$ the Furry distribution emerges whereas for $J \geq 1$ and suitable input parameters the loglog graph shows a linear section representing the Pareto power law behavior (Fig. 1).

\section{Generalized population statistics}

Unfortunately, function (1), when fitted to experimental data, yields very low values of $\bar{N}$ lying usually in the interval $(1,3)$. Earlier measurements [4] of population statistics in air at normal atmospheric conditions indicated a critical value $N_{0} \approx 7 \times 10^{4}$ as a necessary minimum population with which avalanches began to deviate from the Furry distribution. Therefore, the values $\bar{N}$ and $N_{0}$ should be comparable and not so essentially different. The reason for this discrepancy is caused by non-included inner space charges whose electric fields compete with the background field in the discharge gap. The influence of inner space charges cannot be neglected especially in big avalanches with high electron populations. A consequence of action of inner space charges consists in lowering the value of $\alpha$ with increasing electron populations $n[1,5]$ :

$$
\alpha(n)=\alpha_{0}\left[1-B \ln \left(\frac{n}{N_{0}}\right)\right],
$$

$$
N_{0} \leq n \leq 10^{8} .
$$

For air it is anticipated $[1,4] B \approx 0.07, N_{0} \approx 7 \times 10^{4}$ and $\alpha_{0}$ is a constant corresponding to the first Townsend ionization coefficient in the unperturbed background electric field. Dependence (3) represents a typical behavior of the so-called pre-streamer avalanches $n \in\left(10^{4}, 10^{8}\right)$ but at higher populations, $n>10^{8}$, photoionization completely replaces collisional ionization and majority of avalanches are converted into streamers. In the streamer region the value of $\alpha(n)$ quickly restores its initial value $\alpha_{0}$ and increases further $\alpha(n) \gg \alpha_{0}$. However, an exact dependence $\alpha(n)$ is not known for streamers. Nevertheless, for pre-streamer avalanches $\left(\bar{N}=N_{0}\right)$ it is possible to determine a law of growing populations $n(d)$ :

$$
\begin{aligned}
& \mathrm{d} n=\alpha(n) n \mathrm{~d} x, \\
& \int_{\bar{N}}^{n} \frac{\mathrm{d} n}{n\left[1-B \ln \left(\frac{n}{\bar{N}}\right)\right]}=\int_{\bar{\Delta}}^{d} \alpha_{0} \mathrm{~d} x, \\
& n(d)=\bar{N} \exp \left(\frac{1}{B}\left(1-\exp \left(1-B \alpha_{0}(d-\bar{\Delta})\right)\right)\right), \\
& \bar{N} \leq \exp \left(\alpha_{0} d\right) \leq 10^{8} .
\end{aligned}
$$

Using (6), the mean population $\bar{n}_{j}$ of the displaced avalanches of the $j$-th generation can be determined

$$
\begin{aligned}
& \bar{n}_{j}(d) \\
& \quad=\bar{N} \exp \left(\frac{1}{B}\left(1-\exp \left(1-B \alpha_{0}(d-(j+1) \bar{\Delta})\right)\right)\right) \\
& =\frac{\bar{c}}{\bar{A}^{\bar{M}^{j+1}}},
\end{aligned}
$$

where

$$
\begin{aligned}
& \bar{c}=\bar{N} \exp \left(\frac{1}{B}\right) \\
& \bar{A}=\exp \left(\frac{1}{B} \exp \left(1-B \alpha_{0} d\right)\right), \\
& \bar{M}=\exp \left(B \alpha_{0} \bar{\Delta}\right)=\bar{N}^{\mathrm{B}} .
\end{aligned}
$$

Equation (7) enables to formulate a new generalized probability density function which takes into account the influence of inner space charges

$$
\begin{gathered}
F(n)=\frac{1}{\bar{c}} \sum_{j=0}^{J} \bar{K}^{j} \bar{A}^{\bar{M}^{j+1}}\left[1-\frac{\bar{A}^{\bar{M}^{j+1}}}{\bar{c}}\right]^{n+1} \\
\approx \frac{1}{\bar{c}} \sum_{j=0}^{J} \bar{K}^{j} \bar{A}^{\bar{M}^{j+1}} \exp \left(-\frac{n \bar{A}^{\bar{M}^{j+1}}}{\bar{c}}\right) .
\end{gathered}
$$

Function (9) is also capable to generate the Pareto power law behavior. Nevertheless, there is an important difference between functions (1) and (9) consisting in interpretation of the parameters $\bar{N}$ and $\bar{A} \bar{M}=\bar{A}^{N^{B}}$. In contrast to function (1) the probability density (9), when fitted to experimental data (Fig. 2 ), provides $\bar{N} \approx 6.8 \times 10^{4}$ in very good agreement with the value $N_{0} \approx 7 \times 10^{4}$ predicted by earlier experiments [4]. This is due to the 


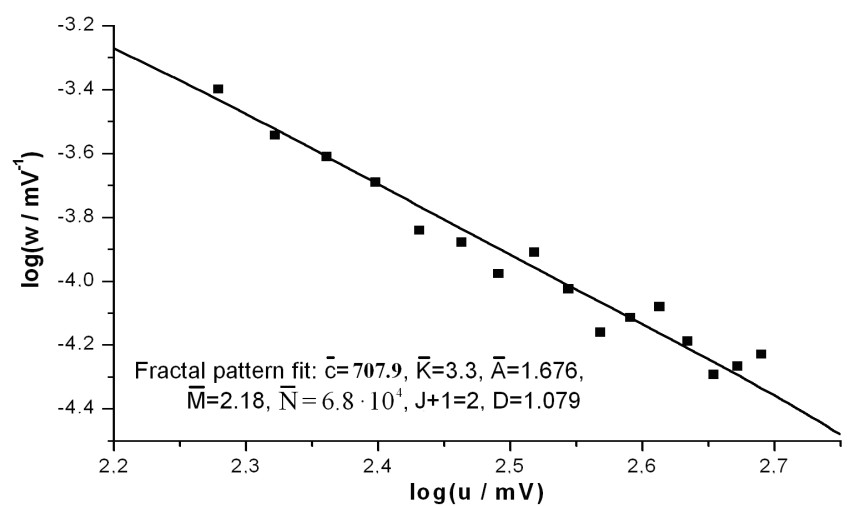

Fig. 2. Population statistics registered as voltage pulses across a resistance $(100 \mathrm{k} \Omega)$ associated with the UV-photomultiplier.

included action of the inner space charge field that interferes with the background field in the gap and as a result the total field between the electron head and ion tail becomes weaker which causes lowering of $\alpha(n)$ values (3). As a consequence, the electron population $n(d)$ grows "under-exponentially" (6) which inevitably modifies the statistics (1) and leads to a new analytical form (9). Although both forms (1) and (9) are probability density functions that include the Furry and Pareto statistics as two special cases $(J=0$ and $J \geq 1)$, only the generalized form (9) provides a right description of statistical behavior of pre-streamer avalanches and becomes an adequate representative of the pre-streamer statistics.

\section{Conclusion}

Probability density function (9) provides a rigorous solution of the long-lasting problem concerning anomalous avalanche statistics, which were considered earlier as ex- perimental artifacts [1]. Furthermore, function (9) generates the fractal power law behavior by means of a superposition of many elementary functions, which may support the concept of multi-componential nature of fractal phenomena.

\section{Acknowledgments}

Support for this work has been provided by GA ČR project $202 / 07 / 1407$.

\section{References}

[1] H. Raether, Electron Avalanches and Breakdown in Gases, Butterworths, London 1964.

[2] L.B. Loeb, Basic Processes of Gaseous Electronics, University of California Press, Berkeley 1960.

[3] J.M. Meek, J.D. Craggs, Electrical Breakdown of Gases, Wiley, New York 1978.

[4] L. Frommhold, Z. Phys. 150, 172 (1958) (in German).

[5] K. Richter, Z. Phys. 180, 489 (1964) (in German).

[6] W.H. Furry, Phys. Rev. 52, 569 (1937).

[7] R. Wijsman, Phys. Rev. 75, 833 (1949).

[8] T. Ficker, J. Appl. Phys. 78, 5289 (1995).

[9] T. Ficker, IEEE Trans. Dielectr. Electr. Insul. 10, 689 (2003).

[10] T. Ficker, IEEE Trans. Dielectr. Electr. Insul. 10, 700 (2003).

[11] T. Ficker, J. Phys. D, Appl. Phys. 40, 7720 (2007).

[12] T. Ficker, IEEE Trans. Plasma Sci. 36, 1310 (2008).

[13] T. Ficker, J. Plasma. Fus. Res. Ser. 8, 744 (2009). 